\title{
Circuit
}

Musiques contemporaines

\section{Les Vêpres de la Vierge (Tremblay)}

\section{Gilles Tremblay}

Volume 6, numéro 1, 1995

Tremblay/Varèse/Messiaen : Gilles Tremblay analyste

URI : https://id.erudit.org/iderudit/902121ar

DOI : https://doi.org/10.7202/902121ar

Aller au sommaire du numéro

Éditeur(s)

Les Presses de l'Université de Montréal

ISSN

1183-1693 (imprimé)

1488-9692 (numérique)

Découvrir la revue

Citer cet article

Tremblay, G. (1995). Les Vêpres de la Vierge (Tremblay). Circuit, 6(1), 51-56. https://doi.org/10.7202/902121ar

Ce document est protégé par la loi sur le droit d'auteur. L'utilisation des services d'Érudit (y compris la reproduction) est assujettie à sa politique d'utilisation que vous pouvez consulter en ligne.

https://apropos.erudit.org/fr/usagers/politique-dutilisation/
Cet article est diffusé et préservé par Érudit.

Érudit est un consortium interuniversitaire sans but lucratif composé de l’Université de Montréal, l'Université Laval et l'Université du Québec à Montréal. Il a pour mission la promotion et la valorisation de la recherche. https://www.erudit.org/fr/ 


\section{Les Vêpres de la Vierge (Tremblay)}

Notes pour le disque Ariane (AR 136 SCA 500cd) - SNE (54 l C) paru en 1986.

Les Vêpres de la Vierge m'ont été commandées à l'occasion du huit cent cinquantième de la fondation de l'abbaye de Notre-Dame-de-Sylvanès. Ces circonstances président donc à la composition destinée à une architecture où l'acoustique est exceptionnelle, comme en témoigne cet enregistrement réalisé lors de la création, le 20 juillet 1986. L'œuvre est écrite pour chœur mixte, soprano solo et treize instrumentistes (trois flûtes et petite flûte, hautbois, cor anglais, deux trompettes, trombone, contrebasse, orgue positif, trois groupes de percussions métalliques). Elle est dédiée à André Gouzes, dominicain, et aux amis de Sylvanès, qui, à la suite de ses architectes, en font une résonance vivante.

Dans la liturgie des heures, les vêpres correspondent au coucher du soleil. C'est dire l'importance symbolique de la lumière (assimilée au Christ). Une place de choix dans la musique a donc été accordée à son évocation, d'autant plus qu'il s'agit de Vêpres de la Vierge, * une Femme ayant le soleil pour manteau (Apocalypse, 12:1).

La plupart des textes sont en français sauf quelques-uns, comme I'hymne Ave maria stella ainsi que deux des anciennes que j'ai laissées en latin. Pour l'accentuation, c'est la langue parlée qui m'a servi de guide, avec toute la flexibilité que donnent la durée, l'espace, la répétition, les vocalises, le silence, pour intensifier, magnifier un mot, une syllabe, à l'exemple de l'ornementation ou des neumes grégoriens.

Exemplarité du grégorien. Par une beauté qui transcende le temps, il garde, plus que jamais, sa force de sève aujourd'hui. Dans ces Vêpres de la Vierge, j'ai tenté de lui articuler cette époque où, comme à la Renaissance, l'écart de style était déjà très grand. J'ai été émerveillé d'y constater une harmonie et une affinité complémentaires, encore plus grande aujourd'hui qu'avec certains langages du passé, souvent trop directionnels par rapport à la modalité. (Je pense notamment au déterminisme d'une sensible se résolvant fatalement sur la tonique.) Ce contact a été un choc enthousiasmant. J'y ai découvert une coexistence non seulement fraternelle et naturelle, mais familière également, comme à la suite d'une longue préparation plus ou moins consciente. 


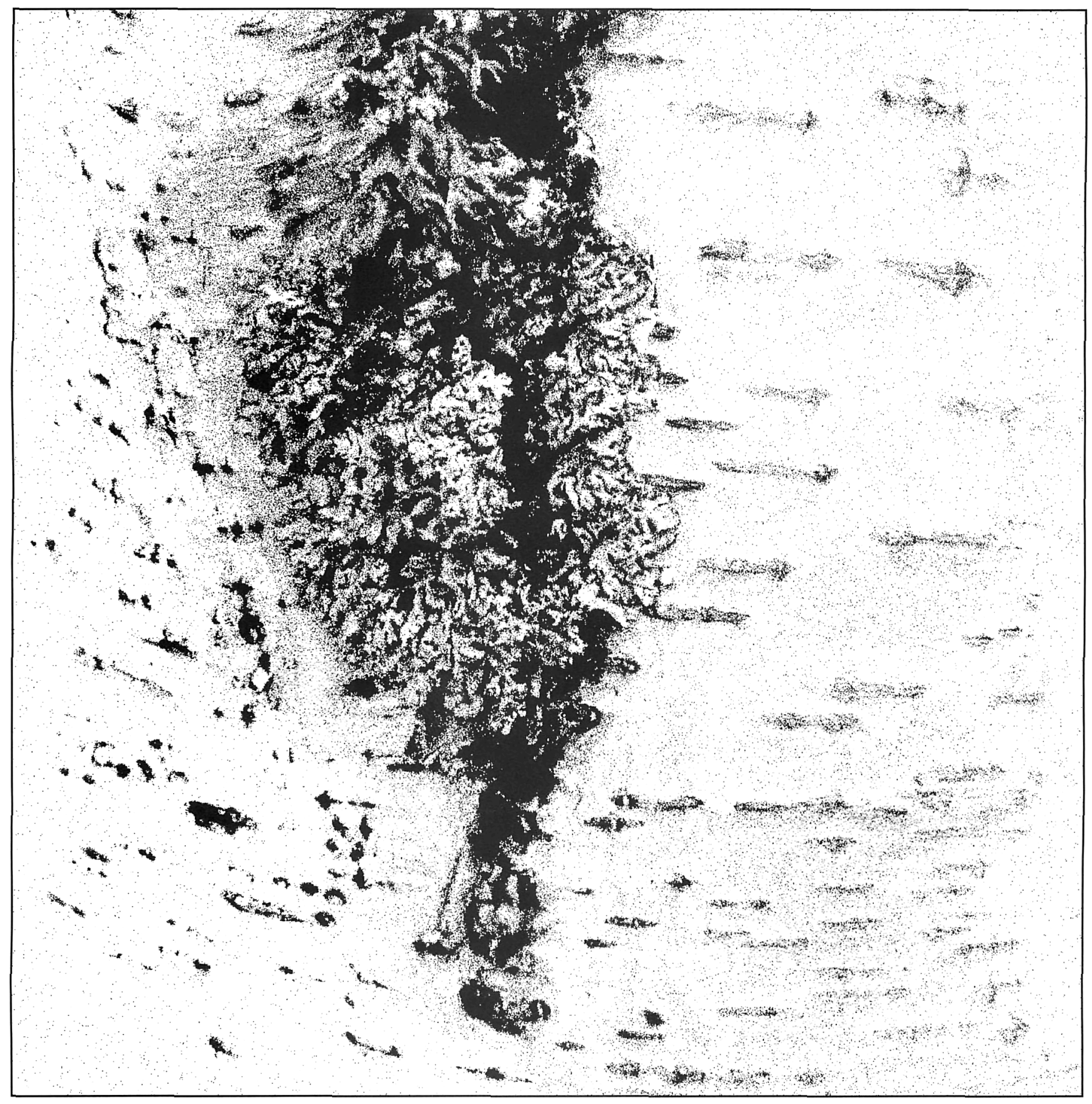


Voici à titre indicatif quelques exemples du type d'articulation utilisé entre les styles: 1) champs-groupes communs se greffant les uns aux autres, servant de plaque tournante d'un style à l'autre ;

Exemple 1

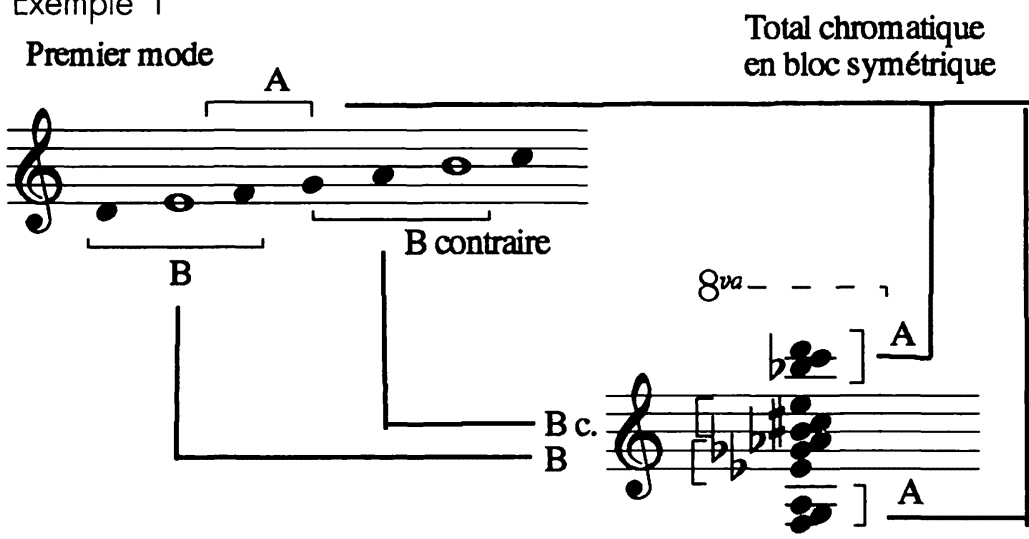

2) ornementation d'une note par un éclat en faisceau ;

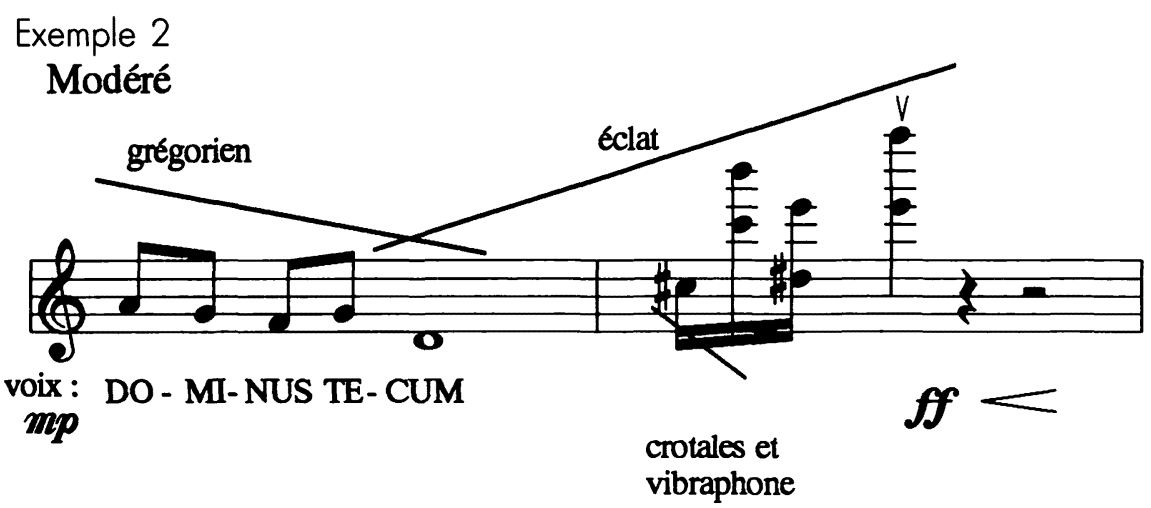

3) mélisme ornemental autour d'une note commune ;

Exemple 3

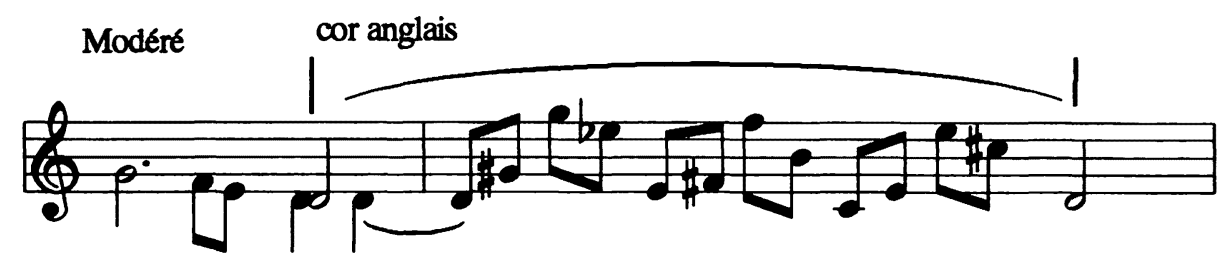

voix : A - lle - lu - ia 
4) rime mélodique commune dans deux styles différents ;

\section{Exemple 4}

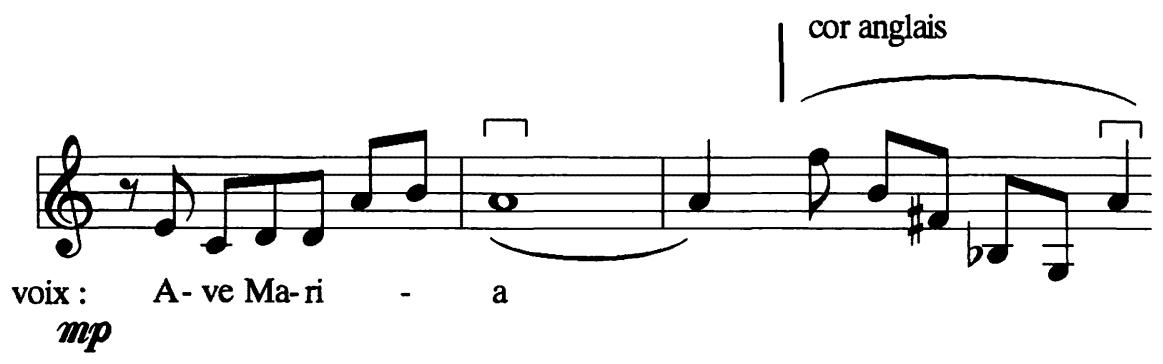

5) amplification d'un son par ses harmoniques naturelles, comme sur le sol du mot FIAT (Antienne II) ;

6) groupes complémentaires entre les sons de la psalmodie modale et les vibrations des métaux aigus donnant le total chromatique harmoniquement articulé, en une constellation de durées-résonances, c'est-à-dire où les intensités variables déterminent les durées, elles-mêmes litaniques.

Tel a été ce défi : citer, enchâsser, souligner, commenter, enluminer, en un jeu d'échanges où les éclairages mutuels se rafraîchissent l'un l'autre, découvrant d'étonnants accents communs, provoquant ce que j'appellerais un effet de sève réciproque d'où est exclue, est-il besoin de le préciser, toute nostalgie passéiste.

Ces vêpres, qui suivent le déroulement liturgique, se divisent en trois grandes parties:

I. prologue : Envol, alléluia pour flûte seule len sept petites sections : lancés; surabondance I; ruissellement I; surabondance $I I$; ruissellement II ; surabondance III, chant lyrique en rotations amples).

Introduction avec:

- Gloria en deux éléments: 11 un grand drapé mélodico-harmonique; 21 deux blocs harmoniques engendrant des couleurs harmoniques réciproques par leurs cinquième et septième harmoniques respectives, en micro-intervalles - les notes fondamentales fa et si sont les mêmes que celles des cloches de l'Abbaye - les phonèmes de l'alléluia sont développés par répétitions et permutations ;

- Lucernaire, avec antienne-choral aux voix de femmes, éclats instrumentaux et cantillation des versets par les voix d'hommes ;

- Bénédiction du célébrant avec exclamations d'Amen aux voix et instruments ; 
- l'hymne Ave maris stella, en grégorien avec ponctuations à durées croissantes au grand tam-tam.

II. Série de psalmodies précédées et suivies de leurs antiennes grégoriennes enluminées par des tropes. Pendant la psalmodie: constellations très douces de durées-résonances. Le mouvement de balancier des versets est ponctué alternativement par un tam-tam et une cloche accordée (II). On a :

Antienne I Ave Maria, avec tropes instrumentales et vocales.

Psaume 121.

Antienne I variée.

Antienne II: «Voici la servante du Seigneur: FIAT», avec introduction et tropes.

Psaume 126.

Antienne II variée.

Antienne III. Benedicta tu avec tropes et alléluias instrumentaux.

Cantique de saint Paul (Éphésiens I).

Antienne III variée avec alléluia instrumental développé.

III. Lecture brève, tirée de l'Apocalypse de saint Jean, par le célébrant. Fulgurance instrumentale, en éclat. Un très doux alléluia lui répond, fondu à la résonance.

Répons bref. Nouvel Ave Marie grégorien, dansant, d'allure presque populaire, sur trois notes seulement. J'ai orné les retours du Dominus tecum de groupes-traits ultra-rapides variés chaque fois.

Magnificat se déroulant sur trois plans: 1) un énorme bruit métallique en relation avec l'évocation de l'Apocalypse : "Un signe grandiose », frisson de l'univers - le métal s'en faisant le prote-parole minéral; 2) une vocaliseMagnificat, servant d'antienne, par le soprano solo, dont la joie prophétique traverse toute la pièce ; 3 ) le texte même du Magnificat grégorien, comme un écrin méditatif au poème de Marie, dont on ne peut épuiser le sens ineffable tellement il touche à l'infini. J'ai tenté pour ma part d'en traduire la joie si grande qu'elle nous dépasse totalement, jusqu'au drame: sa dimension englobe la réalité de la création et l'espérance la plus inouie.
(1) Ces merveilleuses cloches trouvées à Hérépian (Hérault) chez le fondeur François Grasnier.

TREMBLAY, Gilles, Les Vêpres de la vierge, Ariane (AR 136 SCA 500 cd) SNE (54 I C), 1986. 


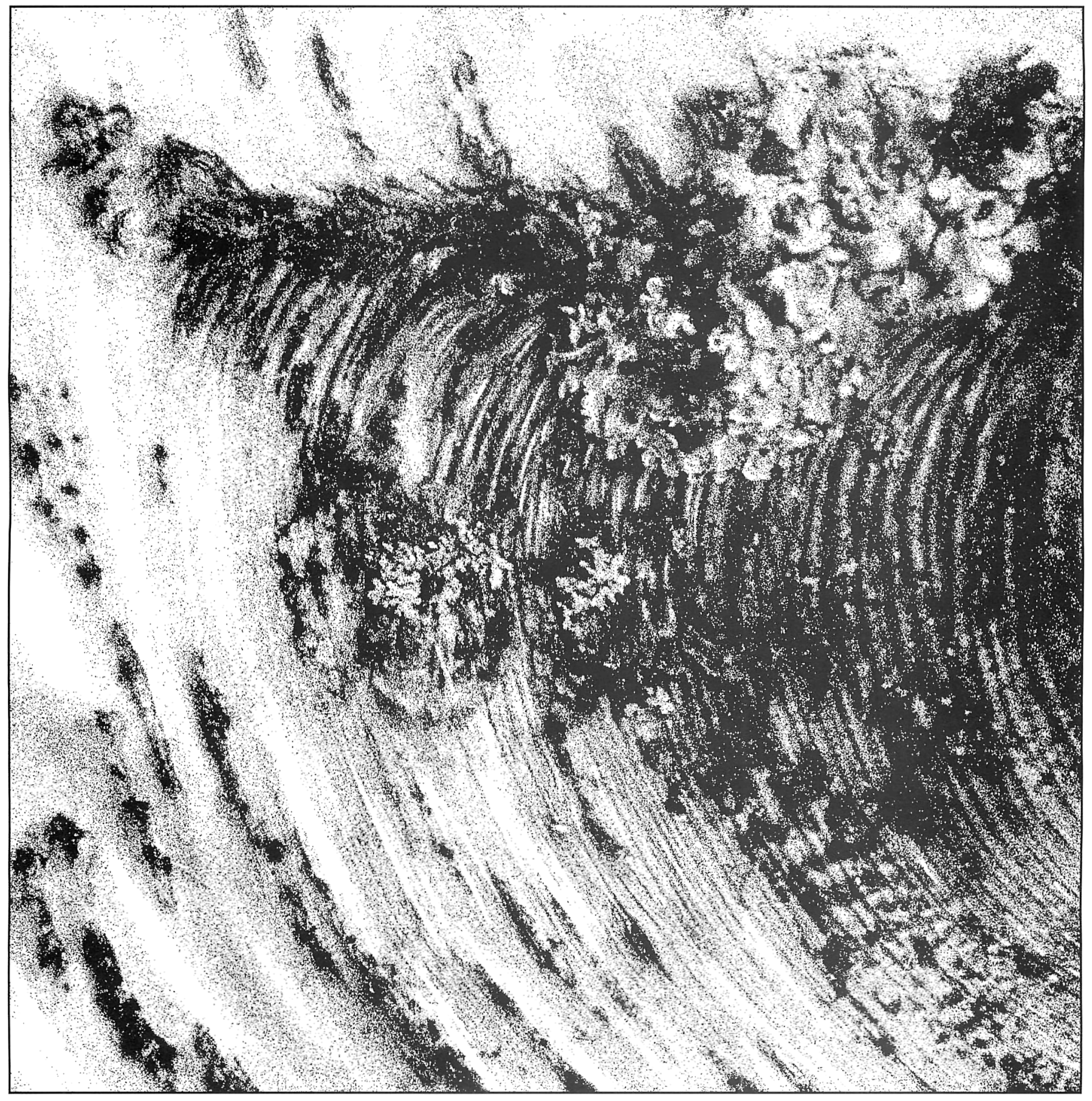

